\title{
CIENCIAMATRIA
}

Revista Interdisciplinaria de Humanidades, Educación, Ciencia y Tecnología

Año VII. Vol. VII. N¹2. Enero - Junio. 2021

Hecho el depósito de ley: pp201602FA4721

ISSN-L: 2542-3029; ISSN: 2610-802X

Universidad Nacional Experimental Francisco de Miranda (UNEFM). Santa Ana de Coro. Venezuela

Juan Andrés Palomeque-Cabrera; Jorge Edwin Ormaza-Andrade;

Guido Olivier Erazo-Álvarez

DOI $10.35381 / \mathrm{cm} . v 7 i 12.436$

\section{La Responsabilidad Social en la Dirección de Movilidad del Gobierno Autónomo Descentralizado de Azogues, Ecuador}

\section{Social Responsibility in the Mobility Department of the Decentralized Autonomous Government of Azogues, Ecuador}

\author{
Juan Andrés Palomeque-Cabrera \\ juanandres.palomeque@est.ucacue.edu.ec \\ Universidad Católica de Cuenca, Cuenca \\ Ecuador \\ https://orcid.org/0000-0003-0508-1826 \\ Jorge Edwin Ormaza-Andrade \\ jormaza@ucacue.edu.ec \\ Universidad Católica de Cuenca, Cuenca \\ Ecuador \\ https://orcid.org/0000-0001-5449-1042 \\ Guido Olivier Erazo-Álvarez \\ oerazo@ucacue.edu.ec \\ Universidad Católica de Cuenca, Cuenca \\ Ecuador \\ https://orcid.org/0000-0002-2494-0967
}

Recibido: 01 de octubre de 2020

Aprobado: 15 de diciembre de 2020 


\author{
CIENCIAMATRIA \\ Revista Interdisciplinaria de Humanidades, Educación, Ciencia y Tecnología \\ Año VII. Vol. VII. N¹2. Enero - Junio. 2021 \\ Hecho el depósito de ley: pp201602FA4721 \\ ISSN-L: 2542-3029; ISSN: 2610-802X \\ Universidad Nacional Experimental Francisco de Miranda (UNEFM). Santa Ana de Coro. Venezuela \\ Juan Andrés Palomeque-Cabrera; Jorge Edwin Ormaza-Andrade; \\ Guido Olivier Erazo-Álvarez
}

\begin{abstract}
RESUMEN
La investigación tiene por objetivo analizar la Responsabilidad Social en la Dirección de Movilidad del Gobierno Autónomo Descentralizado de Azogues, Ecuador. La modalidad metodológica empleada es de carácter descriptiva no experimental transaccional, se abordó una población de 70 personas, quienes fueron encuestas en el departamento de matriculación del GAD de la cuidad de Azogues-Ecuador. La Unidad de Matriculación del GAD de Azogues se preocupa por la colectividad siendo socialmente responsables, realizando estudios de medición de la satisfacción del cliente por el servicio que se brinda fomentando un entramado positivo ante la ciudadanía obteniendo resultados alentadores con cerca del $85 \%$ de satisfacción del servicio brindado, además de ello queda una propuesta de puntos a mejorar en el servicio para estar siempre velando por el mismo, estar en constante contacto puesto que la meta de toda institución es la mejora continua.
\end{abstract}

Descriptores: Administración pública; responsabilidad social; participación del público. (Palabras tomadas del Tesauro UNESCO).

\begin{abstract}
The research aims to analyze Social Responsibility in the Mobility Directorate of the Decentralized Autonomous Government of Azogues, Ecuador. The methodological modality used is of a descriptive, non-experimental transactional character, a population of 70 people was approached, who were surveys in the registration department of the GAD of the city of Azogues-Ecuador. The Azogues GAD Enrollment Unit cares about the community being socially responsible, conducting studies to measure customer satisfaction for the service provided, promoting a positive framework for citizens, obtaining encouraging results with about $85 \%$ of customer satisfaction. service provided, in addition to this there is a proposal of points to improve in the service to be always watching over it, to be in constant contact since the goal of every institution is continuous improvement.
\end{abstract}

Descriptors: Public administration; social responsibility; audience participation. (Words taken from the UNESCO Thesaurus). 


\section{INTRODUCCIÓN}

El momento que se habla de la responsabilidad social dentro de nuestro ámbito no se encuentra explotado, tampoco indagado como debería de ser para tener un rescate adecuado de la temática, considerando que es un tema amplio que abarca la responsabilidad de las entidades autoritarias, administrativos, atención al cliente con o también desde el punto de vista del usuario. Adquiriendo así para las personas que laboran en esta entidad un vacío para dar un nivel satisfactorio al usuario. La sociedad como tal tiene un impacto de responder o quedar en dudas las razones por las que no se toca hace nada para mejorar el servicio en todas las entidades y mucho más las Dirección de Movilidad Del Gobierno Autónomo Descentralizado de la Ciudad de Azogues Ecuador.

Recordemos que en varias entidades públicas como privadas ya se tiene implementado el sistema de calificación de atención al usuario donde se pueda obtener cifras significativas del nivel de apertura que brinda el personal, así teniendo evidencia para capacitar, fortalecer, las características que están siendo observadas por el usuario, brindando a la sociedad un servicio de calidad y calidez. (Gonzáles-Sotelo \& NaranjoAfricano, 2017).

Todo lo antes mencionado está indicado para todos los grupos de interés o stakeholders, siendo estos los más afectados de forma negativa o positiva, ya que en esta instancia es donde se toma las decisiones por parte de las diferentes entidades públicas o privadas empezando desde una pequeña empresa de emprendimiento hasta llegar a las instituciones que sobresalen ante la sociedad y son las más conocidas ya sea por tener buen marketing o por sus ofertas de interés a la población, garantizando de esta manera las necesidades que tiene cada empresa. Así sustentando la rentabilidad y el desarrollo de cada uno de los puestos que tiene una empresa en general.

Tenemos que considerar que la Responsabilidad Social Empresarial (RSE), no solo aplica a las entidades públicas, de lo contrario debe ser establecida y sacar niveles de 


\section{CIENCIAMATRIA \\ Revista Interdisciplinaria de Humanidades, Educación, Ciencia y Tecnología \\ Año VII. Vol. VII. N¹2. Enero - Junio. 2021 \\ Hecho el depósito de ley: pp201602FA4721 \\ ISSN-L: 2542-3029; ISSN: 2610-802X \\ Universidad Nacional Experimental Francisco de Miranda (UNEFM). Santa Ana de Coro. Venezuela \\ Juan Andrés Palomeque-Cabrera; Jorge Edwin Ormaza-Andrade; \\ Guido Olivier Erazo-Álvarez}

impacto para el progreso y desarrollo de la empresa, siendo las pioneras para poder dar un gran ejemplo a las entidades privadas que no garantizan un desarrollo adecuado para la sociedad (Cuevas-Moreno \& Rodrigues-Minor, 2017).

Ecuador es un país con recursos establecido para el sector público, sin considerar que existen entidades privadas que tienen gran cantidad de personal para atención como cartera de negocio, enmarcando que nuestro país al tener in binomio de empresas públicas y privadas ya sea de índole nacional o extranjero, se considera de gran importancia y de impacto los Gobiernos Autónomos Descentralizados (GAD) siendo esta entidad la encargada de dar cumplimiento a las funciones de cada provincia donde el decreto de ordenanza está enmarcada en los artículos aprobados, para poder seguir teniendo vigencia los servicios que ofertan con o una satisfacción al usuario que acide a las instalaciones.

Dentro de esta temática se ha considerado de importancia dar una reseña de al estudio del GAD de la ciudad de Azogues, teniendo como referencia que su creación fue dada el 21 de agosto del año 2014, dando cumplimiento con las funciones y competencias establecidas dentro del marco de objetivos a cumplir mediante la ley orgánica de transporte terrestre, tránsito y seguridad vial, siendo la encargada de la vialidad de la ciudad, regulación y control de actividades, operaciones de transporte terrestre tanto urbano como Inter cantonal, sin desmerecer el control del uso de la vía pública, teniendo gran apertura y un número superado a los 20.000 tipos de transporte anualmente que pasan por el control de matriculación vehicular, identificando que las personas que realizar cualquiera de los servicios no son solo oriundos de la provincia del Cañar sino también de otras provincias, con esto aumentando la responsabilidad del personal en atención a cada uno de los servicios que brinda el Gad de la ciudad de Azogues. (Ilustre Municipalidad de Azogues Alcaldia, 2020).

Dentro de lo establecido se podría tener datos específicos para poder medir el nivel de impacto que tiene el usuario al recibir el servicio en las instalaciones del lugar de estudio, 


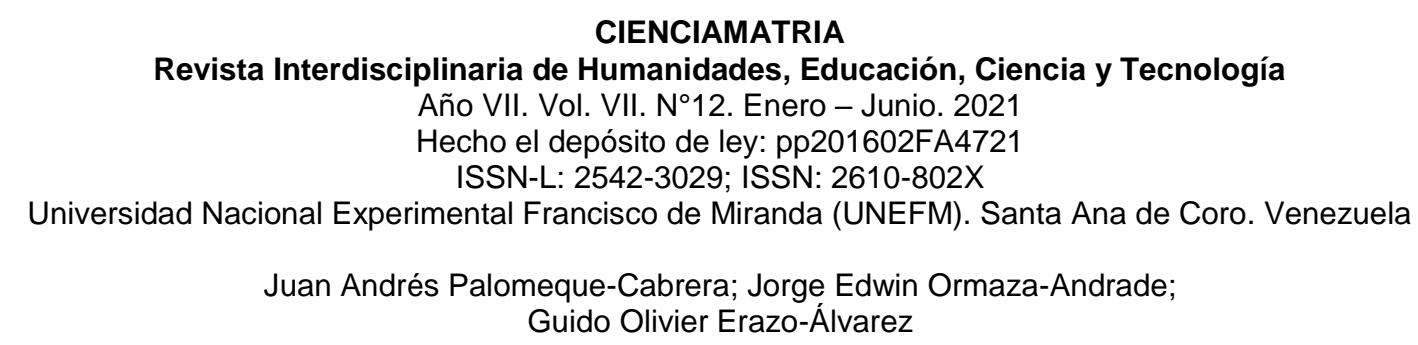

tomando en cuenta que tendríamos datos reales y precisos no solo de los usuarios de la ciudad de Azogues, sino siendo extenso el universo al sacar el muestreo de la localidad de los usuarios de donde acuden para realizar sus diferentes tramites. Con esto se llegaría a tomar medidas correctivas de la atención y servicio al usuario para poder contribuir con la satisfacción del servicio que se brinda quizás en estos momentos no existe una mirada amplia, pero si nos ponemos a revisar la cantidad de usuarios que pedirían algunas acotaciones se demostrara que es necesario dar cumplimiento a esta problemática que afecta a los usuarios como al personal que brinda sus servicios profesionales.

Con esta tabulación de datos utilizando un instrumento recolector de datos específicos donde se pueda tener el nivel de satisfacción del usuario ante el servicio que brinda el GAD de la ciudad de Azogues, logrando tener resultados reales para poder realizar propuesta de mejoras ya sea con capacitaciones o implementación de protocolos que podrán servir para mejorar el servicio al usuario de la ciudad de Azogues como de las diferentes ciudades y provincias, demostrando mediante esta cuantificación de resultados un impacto para la ciudadanía en general y que se pueda dar en todos los GAD.

En tal sentido, la investigación tiene por objetivo analizar la Responsabilidad Social en la Dirección de Movilidad del Gobierno Autónomo Descentralizado de Azogues, Ecuador

\section{Referencial teórico}

\section{La responsabilidad social en los servicios brindados por las entidades públicas.}

Se empezará definiendo el significado de responsabilidad que es el cumplimiento de las obligaciones de manera correcta mediante los valores éticos y morales de las personas, además del cuidado al tomar decisiones al momento de realizar cualquier tipo de actividades de algo específico o de alguna persona (Pérez, 2020).

Además, se habla de la capacidad existente en todo sujeto activo de derecho para reconocer y aceptar las consecuencias de un hecho que se lo ha realizado libremente. 


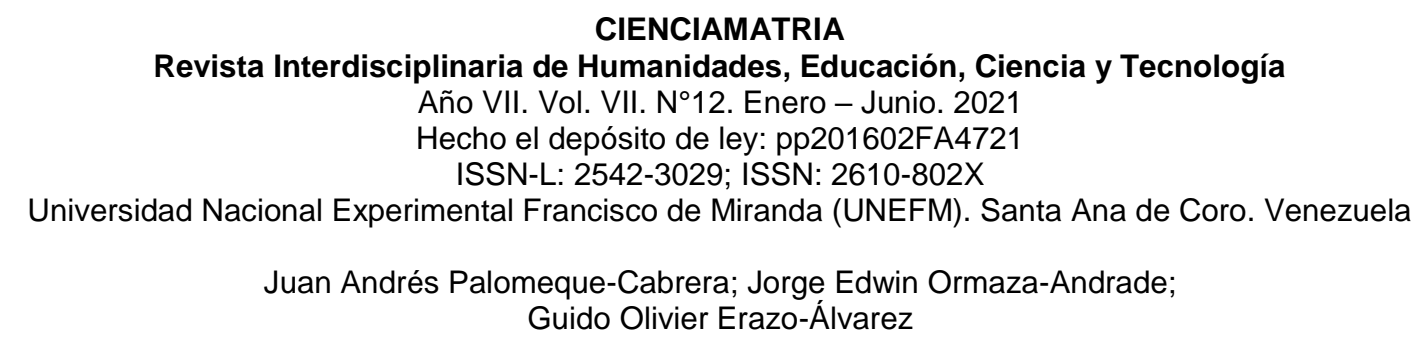

Algunas personas la consideran como una cualidad y un deber moral puesto que influye en muchas repercusiones por las acciones de ser responsables, aquello no radica únicamente en las personas, sino en toda la sociedad, empresas, entidades públicas y más. Con ello nos lleva a la responsabilidad civil que la cual trata acerca de los daños y de la responsabilidad en reparar lo causado, se tiene presente el tema legal por los daños causados a partir de las leyes que se encuentren vigentes o a un contrato previamente establecido (Pérez, 2020).

Pues bien (Correa-Jaramillo, 2007), habla acerca de la responsabilidad social pública que es un tema que tiene su auge en los años 1990 y que hoy en día está presente en los debates a nivel mundial, lo cual contribuye al progreso de la sociedad puesto que se encuentra inmersa la ética, transparencia y la participación de la ciudadanía. Las entidades públicas deben ser socialmente responsables, deben ejercerla y además promoverlas como ejemplo puesto que sus acciones repercuten dentro del grupo de intereses que posean.

Para (Gonzáles-Sotelo \& Naranjo-Africano, 2017), la responsabilidad institucional pública (RIP) es equivalente a la responsabilidad social empresarial del sector privado en las cuales otorgan bienes o servicios a las distintas necesidades de la colectividad y tiene su impacto ante la forma que se administran. La responsabilidad institucional pública debe actuar con equidad social, además del cumplimiento de leyes y estándares a nivel colectivo puesto que se relacionan con centenares de grupos humanos.

La responsabilidad empresarial (RSE) la podemos decir responsabilidad social corporativa la cual engloba los temas descritos anteriormente, como el compromiso voluntario que tiene una empresa para con la sociedad y el medioambiente que la rodea, puesto que las empresas por medio de las relaciones humanas crean vínculos con la sociedad, con sus grupos de intereses con el cual les sirve para mejorar su promoción, venta de servicios y productos (Prieto, 2015). Con ello la responsabilidad social es el 


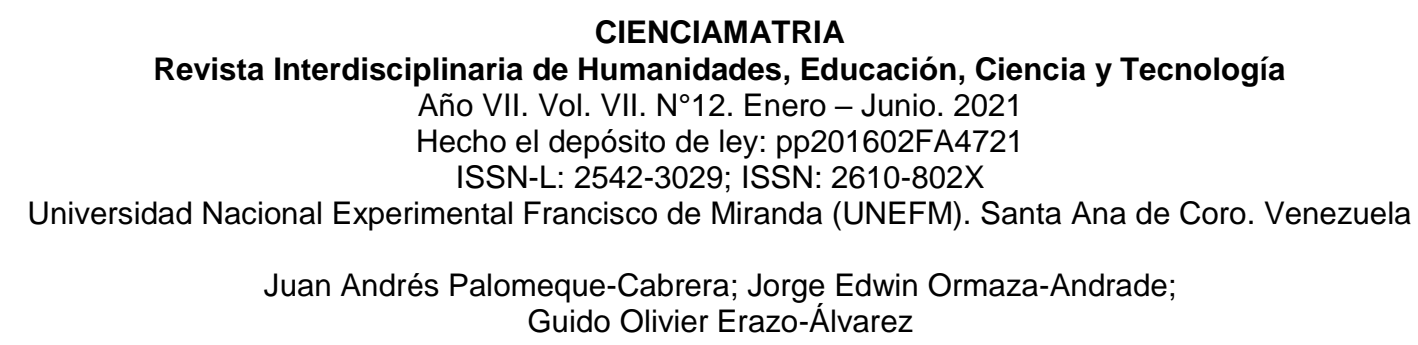

compromiso que tienen los miembros de una sociedad encaminada en las normas de la entidad correspondiente y a la sociedad en sí.

La responsabilidad social de las entidades públicas las cuales son objeto de debate puesto que estas están conformadas mediante la contribución de patrimonio público a lo largo del tiempo teniendo un vínculo estrecho con los grupos de interés además de algunas que se han independizado y son autónomas, en las cuales está latente la responsabilidad social y ambiental para con la sociedad, las cuales deben velar por el desarrollo sustentable, mejora continua y trato de calidad a sus contribuyentes (Fernández, 2016).

Al ser entidades públicas no quiere decir que estén inmersas en la responsabilidad social, velar o trabajar por sus grupos de interés, o mediante la eliminación de los aspectos negativos de sus servicios. La sociedad es la que debe mostrar preocupación por procesos los cuales no se los esté llevando de la manera correcta y eficiente. Según (Fernández, 2016), habla sobre las dificultades que las empresas públicas presentan, como es el caso de la corrupción que está en auge en la última década, además la burocracia de la gestión ante el compromiso político, todo esto al administrar recursos de los cuales cuyo último propietario se encuentra distante al tomador de la decisión.

Por otro panorama esta no es la realidad de todas las entidades públicas puesto que algunas se manejan con total transparencia, los recursos manejan de forma eficiente, preocupados por la sociedad y todo esto se complementa con rendición de cuentas de las gestiones que se han desarrollado a lo largo de la temporada. Deben existir iniciativas por parte de las entidades públicas y privadas en su administración al incorporar prácticas de responsabilidad social con sus grupos de intereses; realizar informes públicos de desempeño económico, social y ambiental ante la colectividad (Fernández, 2016).

Las entidades públicas deben ser transparentes y velar por los ciudadanos que son los mandantes y son pieza fundamental para el desarrollo y sustento de las entidades. No obstante, la adaptación de la responsabilidad social al ámbito público plantea a priori la 
necesidad de dirimir entre su voluntariedad y obligatoriedad considerando que el tratamiento no puede ser idéntico en relación al sector privado y empresarial, de modo que la adopción voluntaria de estrategias socialmente responsables en la gestión empresarial no puede extrapolarse a la administración donde el nivel de exigencia debiera ser superior (Ruiz-Rico Ruiz, 2014).

Desde el punto de vista práctico, tomando como referencia (Cuevas-Moreno \& Rodrigues-Minor, 2017), la responsabilidad social adquiere relevancia por las implicaciones sociales y políticas de la gran empresa y el Estado generan sobre el organismo social. Además, así, la calidad en la gestión pública promueve una cultura transformadora que impulsa a la mejora permanente, con el fin de satisfacer de manera oportuna y adecuada las necesidades y las expectativas de la ciudadanía. La gestión pública y los conceptos de calidad pretenden la mejora continúa siendo preocupados por la satisfacción al cliente y que aquello genere confianza en los mismos (Ministerio del Trabajo, 2020).

Esto sería lo idóneo por parte de las entidades públicas, pero no es la realidad de todas, al ser públicas muchas de ellas no miden la satisfacción del cliente o están pendientes de la mejora continua que sería parte fundamental de toda empresa privada que se preocupe por sus puntos de atención número 1 que son sus consumidores. (AndreaTrujillo \& Vélez-Bedoya, 2006), mencionan que la responsabilidad social constituye representa un transporte estratégico para la empresa con el cual son compatibles las expectativas y los intereses de los accionistas y stakeholders. Con lo mencionado las empresas además de desenvolverse en lo económico, lo hacen en el ámbito social y ambiental en el cual pueden ocasionar impactos negativos, al momento de realizar cambios al sistema o velar por intereses particulares, dejando al pueblo a un costado. Los grupos de interés o stakeholders, son aquellos que tienen interés en alguna empresa en común o se encuentran inmersos en las operaciones que estas realicen, además se encuentra la ética comercial, la salud y seguridad como áreas esenciales para los grupos 


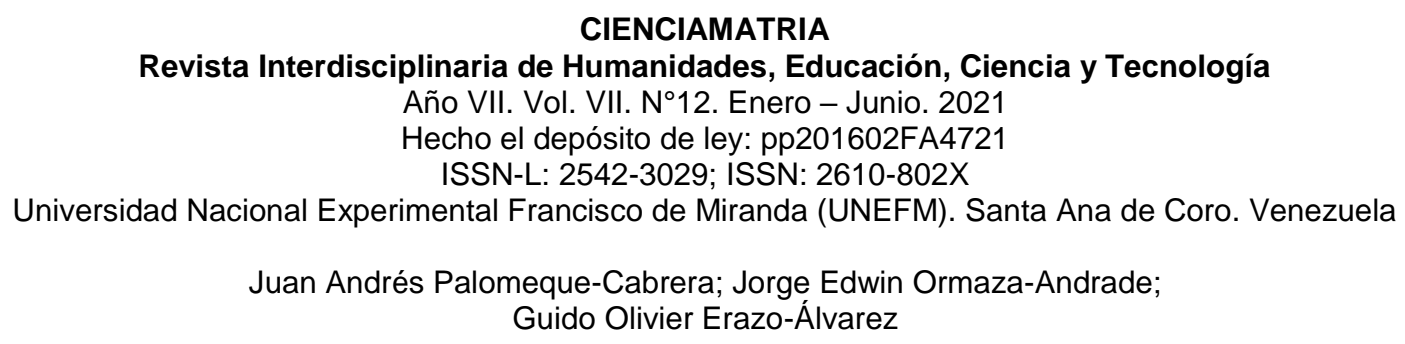

de interés orientados hacia la comunidad. Por medio de opiniones respecto a determinadas acciones influyen hacia las empresas $u$ organizaciones. Gracias a la organización de las Naciones Unidas, las empresas se comprometen con los derechos humanos, el comercio de forma justa y el compromiso de precautelar en el medioambiente que se desenvuelvan las mismas (Prieto, 2015). Siguiendo con este autor, los grupos de interés en cuanto respecta a las administraciones públicas, esperan que se cumplan las leyes vigentes que se rijan en dichas administraciones además del dialogo y establecimiento de normas que mejoren la calidad en el uso de los servicios para con los ciudadanos.

Mediante los diez principios del pacto mundial de naciones unidas de derechos humanos, menciona que "las empresas deben asegurarse de no ser cómplices en abusos de los derechos humanos". En Ecuador el Instituto de responsabilidad social empresarial (IRSE) siendo una organización privada impulsa la práctica y acciones para la implementación de la responsabilidad social.

A su vez debemos mencionar que existen clientes internos y externos dentro del ámbito organizacional y empresarial, los clientes internos son los empleados de la empresa aquellos que prestan sus servicios. Cliente externo se lo define como aquellos hacia los que están orientados los productos y servicios de las empresas o entidades públicas, siendo los antes mencionados los que proporcionan el flujo de ingresos hacia la empresa (Moreno \& Bernal, 2014).

Por el hecho de utilizar los servicios y generar réditos hacia las empresas se escucha a menudo la frase "El cliente siempre tiene la razón" puesto que todo está enfocado hacia lo mismo. Según (Moreno \& Bernal, 2014), el cliente externo es aquel consumidor de un producto, servicio que ofrece la empresa o entidad pública, el cual constituye la fuente de ingreso influyendo en el desarrollo de las organizaciones.

Para el tema de la entidad pública el cliente externo es el contribuyente de las mismas puesto que con el ingreso de impuestos municipales y públicos por parte de los 


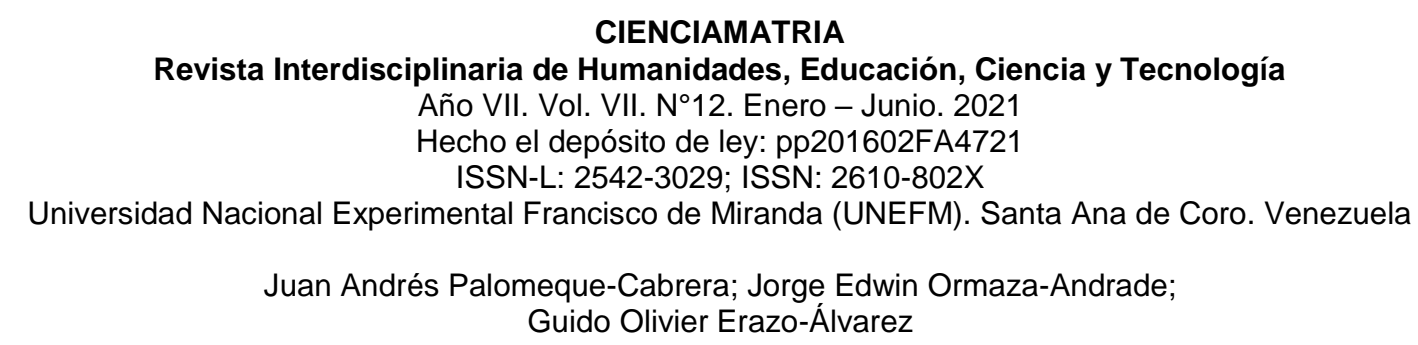

contribuyentes funcionan en parte las entidades públicas, por cual este cliente es de vital importancia y no es tomado como objeto para mejorar o velar por la satisfacción del mismo al momento de utilizar los servicios, puesto que los usuarios deben satisfacer sus necesidades como son: ser cordialmente atendidos, comprendidos, escuchados y dar soluciones a sus requerimientos.

Según (Thompson, 2005), la satisfacción al cliente radica en ocupar un espacio dentro de la mente de los clientes, manteniéndolos satisfechos y siendo el principal objetivo de las empresas, resulta de comparar el rendimiento percibido de un producto o servicio con sus expectativas. Con esto es primordial que las personas que forman parte de las empresas, entidades públicas tengan conocimiento de los beneficios de lograr la satisfacción del cliente y a su vez encontrarse capacitados para cumplir las expectativas de los mismos. Existen réditos al hablar acerca de la satisfacción del cliente como lo son: lealtad hacia la marca, entidad o empresa, generar un mensaje positivo obteniéndose un plus para la empresa y además elevar la participación dentro del mercado (Thompson, 2005).

De igual manera siguiendo con este autor se puede mencionar acerca de los factores que conforman la satisfacción de cliente: como lo es el rendimiento percibido, el cual es como percibió el producto o servicio recibido. Ahora las expectativas se las puede llamar como las esperanzas o el anhelo que tienen los consumidores usuarios y finalmente el nivel de satisfacción que se han generado luego de haber hecho uso de los servicios o productos adquiridos, los mismos que pueden ser medidos mediante escalas, donde se puede obtener satisfacción, insatisfacción y complacencia, alcanzando las expectativas, no alcanzando las mismas y excediéndolas en dicho orden a lo percibido del cliente (Thompson, 2005).

Lo mencionado es un tema de realidad un usuario satisfecho comenta su experiencia de lo vivido del trato, del tiempo de atención, etc. Todo esto conlleva a un beneficio mutuo para el cliente o usuario de las empresas como de las entidades públicas al tener 


\section{CIENCIAMATRIA \\ Revista Interdisciplinaria de Humanidades, Educación, Ciencia y Tecnología \\ Año VII. Vol. VII. N¹2. Enero - Junio. 2021 \\ Hecho el depósito de ley: pp201602FA4721 \\ ISSN-L: 2542-3029; ISSN: 2610-802X \\ Universidad Nacional Experimental Francisco de Miranda (UNEFM). Santa Ana de Coro. Venezuela \\ Juan Andrés Palomeque-Cabrera; Jorge Edwin Ormaza-Andrade; \\ Guido Olivier Erazo-Álvarez}

servicios de calidad con lo cual todo aquello deriva en aumentar los ingresos y captación de utilidad de las mismas. En el caso de las entidades públicas lo que generan con la satisfacción al cliente es poner en práctica la responsabilidad social al velar y preocuparse por la ciudadanía al recibir un buen trato puesto que las entidades públicas sin la aportación de la ciudadanía no existieran, debido a lo cual se debería realizar mediciones periódicamente para la mejora continua.

Además de ello es fundamental conocer a los clientes, cuáles son sus necesidades, toda persona merece ser tratada de manera cordial, con una actitud positiva, sentir empatía y apoyo por parte de los funcionarios que brindan su servicio. No obstante, la satisfacción al cliente no queda allí, sino en realizar una medición de la misma refiriéndonos: al servicio, el tiempo de espera y la solución de su problema o requerimiento. Para tocar este tema tenemos algunas variables a examinar que afectan en la satisfacción del cliente, como es el tiempo de espera, la calidad del servicio, el trato en la atención, el desempeño percibido, todas estas tienen su impacto al momento de la satisfacción al cliente.

Según (Albrecht, 1992), menciona que la calidad se la evalúa mediante el servicio brindado para obtener información y con esto mejorar el proceso o sistema que se emplee para poder obtener mejores resultados. Todo esto dicho desde el punto de vista de la satisfacción al cliente es decir realizar encuestas acerca el servicio brindado para la retroalimentación del mismo y con esto plantear nuevas alternativas o cambios en el sistema con el único fin de cumplir con las expectativas de los clientes. Continuando con este autor habla sobre el triángulo de servicios mediante la estrategia de servicio que radica en la prioridad del cliente, la segunda se enfoca hacia el personal operativo orientado al cliente mediante la adecuada capacitación de respuesta y de ayuda a los mismos, la tercera está basada en el trato amistoso hacia el cliente.

Este triángulo desde el punto de vista de la satisfacción al cliente cuenta con el correcto entramado puesto que se debe precautelar el servicio, la respuesta y el trato hacia el 


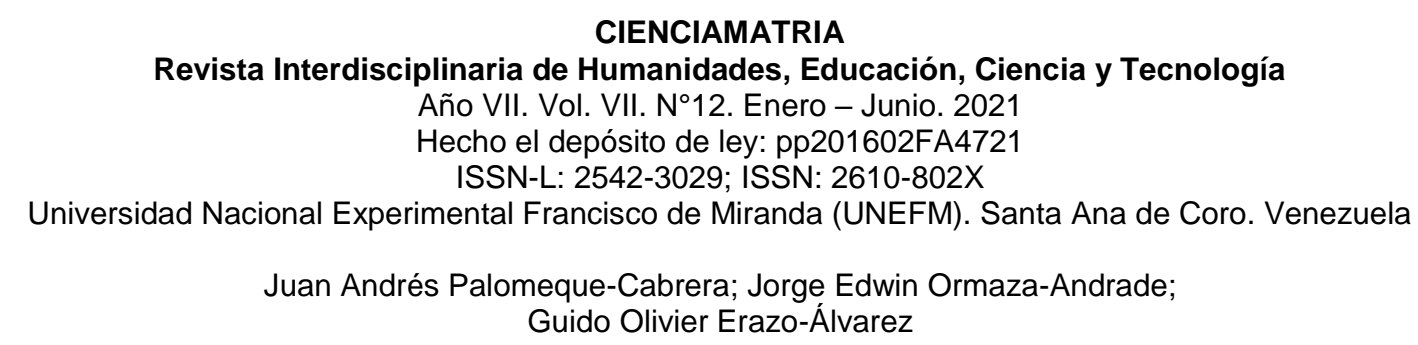

usuario, es decir manejar el adecuado proceso de servicio estar a la vanguardia para cumplir con las expectativas del servicio, tener al personal calificado, orientado hacia el cliente y capacitado para solventar el requerimiento solicitado por parte de los mismos y finalizando contar con personal comprometido a su trabajo para que pueda transmitir la política de la empresa o entidad pública.

Dentro del ámbito de la satisfacción al cliente el tiempo de espera, es una variable influyente, el tiempo es el enemigo de toda índole que sucede al día a día y más aún cuando se requiere de un servicio en la empresa privada como en la pública. Según (Block, et al., 2000), se debe diferenciar dos tipos de tiempos los cuales son: el tiempo real y el tiempo percibido los dos tienen diferentes impactos dentro de los usuarios al momento de realizar un trámite u optar por un servicio.

El modo de ver al tiempo depende de factores internos cuando son propios del usuario y ajenos al servicio que se requiera utilizar en ese momento tales como lo son: estado de ánimo de las personas, el nivel del cansancio con la que cuenten y los externos los cuales son derivados del servicio que se pueden controlar y todos aquellos a su vez influyen en la satisfacción del cliente (Alvarado-Valencia \& Trespalacios-Leal, 2016).

La calidad del servicio es un punto clave que se debe tener en cuenta en toda empresa, que se debe cumplir sin importar el tamaño, estructura y naturaleza de sus operaciones, con la cual se demuestra la capacidad de la misma para cumplir con servicios y funciones demandadas por los clientes. (Aceves-López \& Solórzano-Barrera, 2013). Ahora pues bien, las empresas privadas que venden servicios y productos están enfocadas en la atención, el cumplimiento de expectativas de los clientes para poder seguir en el mercado y generando utilidad, en cambio en las entidades públicas no sucede lo mismo puesto que el servicio es gratuito y no existe competencia para cautivar a los usuarios que obtén por su servicio y es ahí donde entra la responsabilidad social por parte de las entidades públicas para ofrecer un sistema de calidad. 


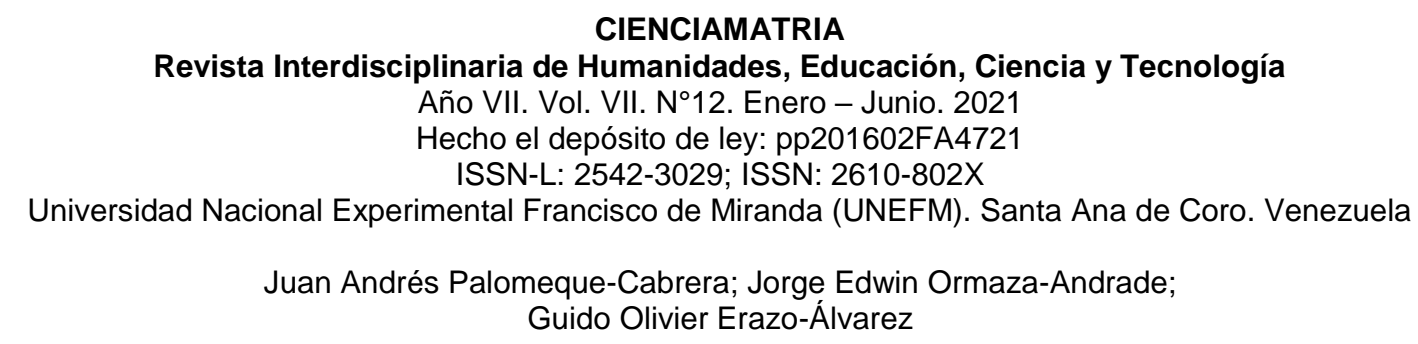

Es decir, si se ofrece un sistema de calidad por parte de las entidades públicas los usuarios se van satisfechos y esto genera una buena imagen para las entidades y el cumplimiento positivo con sus demandantes. Pues bien (Aniorte, 2020), menciona algunas de las características que se deben cumplir para un servicio de calidad como lo son: el cumplimiento de objetivos, los servicios deben ser adecuados para su uso, brindar solución a sus necesidades y por último proporcionar resultados alcanzados. Con todo esto la satisfacción al cliente se ve reflejada en la calidad, cantidad, precio y tiempo del servicio o producto. En las instituciones públicas es diferente y lo que se busca es un trato justo, cordial que le puedan ayudar y brindar solución a sus requerimientos y sumados a estos el tiempo que demora el servicio (Lazo-Torres, et al. 2019).

Por otro lado, (El Ministerio del Trabajo, 2020), menciona dimensiones acerca de la calidad de servicios que se los divide en: tangibles donde se hace énfasis en la infraestructura, señalética y equipamiento; y las intangibles como lo son la seguridad, confiabilidad, capacidad de respuesta y empatía. Según (Vartuli, 2008), logrando la satisfacción al cliente se consigue beneficios para la empresa o entidad pública entre algunos de ellos como lo son: menores quejas y reclamos por parte de los usuarios, se consigue mostrar una mejor imagen empresarial, se logra un mejor clima laboral puesto que todos están comprometidos con sus funciones que desempeñan.

Ahora pues bien el rendimiento percibido puede definirse como la entrega de valor, el empeño que se le ha brindado a su requerimiento mediante la percepción del usuario. Este rendimiento percibido tiene características tales como: la apreciación del producto o servicio que se requiere por parte del usuario, el rendimiento no se basa en una realidad objetiva sino subjetiva y además el estado anímico del usuario o cliente influye en la percepción al igual que los comentarios de terceras personas (Efiempresa, 2017).

Además cabe acotar una breve reseña del "Manual de AMA para la satisfacción al cliente", en el cual hace mención al "Premio Nacional a la Calidad Malcom Baldrige", este es un compromiso a la calidad por parte del Departamento de Comercio de los Estados 
Unidos, este premio se reconoce a las compañías que sobresalen a la calidad de sus productos, servicios y administración en la cual su criterio a medir radica en 7 categorías donde la satisfacción al cliente es la consideración más importante, indicada en la siguiente tabla 1 :

\section{Tabla 1}

Categorías en la medición de la satisfacción al cliente "Malcom Baldrige".

\begin{tabular}{cl}
\hline Porcentaje de importancia & \multicolumn{1}{c}{ Categoría } \\
\hline 30 & Satisfacción al cliente \\
15 & Utilización de los recursos humanos \\
15 & Garantía de calidad \\
15 & Resultados de la calidad \\
10 & Liderazgo \\
9 & Planeamiento de las calidades estratégicas \\
6 & Información y análisis
\end{tabular}

Fuente: Dutka \& Mazia (1994).

Siguiendo con este "Manual de AMA para la satisfacción al cliente" nos indica que mediante la satisfacción al cliente sirve además para: conocer las demandas y expectativas de los mismos, el desarrollo de pautas de servicio, la medición del grado de satisfacción, la identificación de tendencias para la innovación con la competencia.

Siguiendo con la temática las encuestas de satisfacción del cliente se las debe medir mediante escala numérica para cuantificar los resultados. La escala de Likert es un instrumento de medida psicométrico mediante el cual el encuestado afirma estar de acuerdo $o$ en desacuerdo sobre algún tema en específico consultado mediante afirmación, reactivo y escala unidimensional ordenada (Bertram, 2008). 


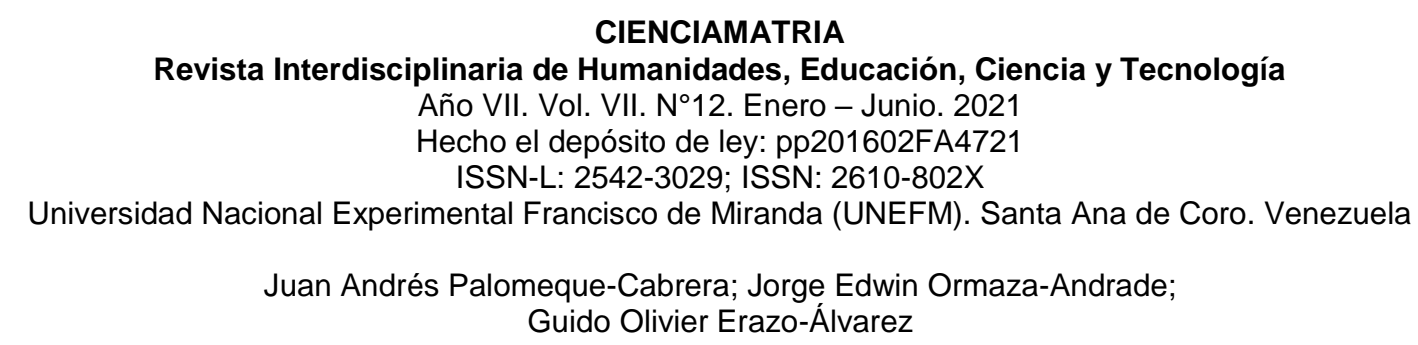

Zagal (2010), menciona que las encuestas de satisfacción a los usuarios se las debe realizar por medio del uso de cuestionarios cortos con el propósito de ser llenados en poco tiempo y de manera objetiva, para el cual se debe seleccionar las preguntas a ser llenadas que partan de los objetivos específicos definidos por parte de la empresa o entidad que lo esté requiriendo.

Se habla también de los indicadores Ethos de Responsabilidad Social Empresarial donde menciona la atención básica, solución de las demandas individuales, proporcionar fácil acceso del consumidor al servicio de su interés, resolver de manera rápida las demandas a partir de los procedimientos adoptados, buscar la causa de los problemas para perfeccionar la calidad de los servicios y finalmente promover la mejora continua en su atención priorizando el dialogo (Instituto Ethos de Empresas e Responsabilidad Social, 2011).

Ahora bien, hasta qué punto es bueno la satisfacción al cliente, es algo que muchos autores discrepan, puesto que para aumentar la satisfacción del cliente es necesario invertir o incurrir en gastos como pueden ser administrativos, de infraestructura, de mercadotecnia, etc. Tocando de parte a las entidades públicas están son diferentes a las empresas privadas puesto que no compiten por brindar sus servicios sino brindan lo que consideran ante la ciudadanía y no se enfocan en la satisfacción de los usuarios más bien se enfocan al cumplimiento normativo interno y estratégico (Calle-Calderon, e al., 2020).

Concluyendo por medio de la satisfacción al cliente la entidad pública se encuentra comprometida con la responsabilidad social de la misma para con sus contribuyentes, además mediante las encuestas para definir la satisfacción al cliente se dará a conocer los procesos y variables que afectan a menor o mayor medida hacia la satisfacción de los mismos, buscando siempre una relación directa entre las opiniones de los usuarios con los datos obtenidos de los indicadores en las encuestas, es decir adoptando el punto de vista del usuario. 
CIENCIAMATRIA

Revista Interdisciplinaria de Humanidades, Educación, Ciencia y Tecnología

Año VII. Vol. VII. N¹2. Enero - Junio. 2021

Hecho el depósito de ley: pp201602FA4721

ISSN-L: 2542-3029; ISSN: 2610-802X

Universidad Nacional Experimental Francisco de Miranda (UNEFM). Santa Ana de Coro. Venezuela

Juan Andrés Palomeque-Cabrera; Jorge Edwin Ormaza-Andrade;

Guido Olivier Erazo-Álvarez

\section{MÉTODO}

En la presente investigación la modalidad metodológica empleada es de carácter descriptiva no experimental transaccional, puesto que el estudio se realizó con el suceso natural de las variables para el respectivo análisis, síntesis y conclusión. Se abordó una población de 70 personas, quienes fueron encuestas en el departamento de matriculación del GAD de la cuidad de Azogues-Ecuador, mediante cuestionario en escala Likert con la finalidad de medir el nivel de satisfacción de los servicios recibidos, siendo validado por juicio de expertos y cálculo de coeficiente Alfa de Cronbach con un resultado de 0,89 siendo considerado confiable para su aplicación, mientras que la información recopilada fue procesada en razón de la estadística descriptiva.

\section{RESULTADOS}

Una vez con las encuestadas realizadas a los usuarios de la Unidad de Matriculación del GAD de Azogues, las mismas que se las realizo de manera física, los resultados obtenidos se los proceso mediante el software de Microsoft Excel con un total de 70 encuestados, para su posterior interpretación se los dividió los resultados en dos dimensiones, para una mejor interpretación de las cuales podemos definir el servicio brindado en la tabla 2 a continuación: 
CIENCIAMATRIA

Revista Interdisciplinaria de Humanidades, Educación, Ciencia y Tecnología

Año VII. Vol. VII. N¹2. Enero - Junio. 2021

Hecho el depósito de ley: pp201602FA4721

ISSN-L: 2542-3029; ISSN: 2610-802X

Universidad Nacional Experimental Francisco de Miranda (UNEFM). Santa Ana de Coro. Venezuela

Juan Andrés Palomeque-Cabrera; Jorge Edwin Ormaza-Andrade;

Guido Olivier Erazo-Álvarez

Tabla 2.

Servicio brindado por parte de la Unidad de Matriculación.

\begin{tabular}{|c|c|c|c|c|c|}
\hline & $\begin{array}{c}\text { Nada } \\
\text { satisfecho }\end{array}$ & $\begin{array}{c}\text { Poco } \\
\text { satisfecho }\end{array}$ & Neutral & $\begin{array}{c}\text { Muy } \\
\text { satisfecho }\end{array}$ & $\begin{array}{l}\text { Totalmente } \\
\text { satisfecho }\end{array}$ \\
\hline Calidad del servicio & $0 \%$ & $5 \%$ & $5 \%$ & $32 \%$ & $28 \%$ \\
\hline $\begin{array}{l}\text { Eficiencia en el } \\
\text { servicio }\end{array}$ & $1 \%$ & $1 \%$ & $8 \%$ & $27 \%$ & $33 \%$ \\
\hline $\begin{array}{l}\text { Organización y } \\
\text { logística en el } \\
\text { trabajo }\end{array}$ & $1 \%$ & $0 \%$ & $4 \%$ & $38 \%$ & $27 \%$ \\
\hline \multicolumn{6}{|c|}{$\begin{array}{l}\text { A continuación, se tabuló de igual manera la dimensión corres } \\
\text { usuario que es aspecto importante dentro de la satisfacción d } \\
\text { detallan a continuación en la tabla } 3 \text { : } \\
\text { Tabla } 3 . \\
\text { Atención del usuario por parte de la Unidad de Matriculación. }\end{array}$} \\
\hline & $\begin{array}{c}\text { Nada } \\
\text { satisfecho }\end{array}$ & $\begin{array}{c}\text { Poco } \\
\text { satisfecho }\end{array}$ & Neutral & $\begin{array}{c}\text { Muy } \\
\text { satisfecho }\end{array}$ & $\begin{array}{l}\text { Totalmente } \\
\text { satisfecho }\end{array}$ \\
\hline Información & & & & & \\
\hline $\begin{array}{l}\text { proporcionada en el } \\
\text { servicio }\end{array}$ & $0 \%$ & $3 \%$ & $1 \%$ & $43 \%$ & $53 \%$ \\
\hline $\begin{array}{ll}\text { Desempeño } & \text { y } \\
\text { desenvolvimiento } & \\
\text { del profesional } & \end{array}$ & $0 \%$ & $3 \%$ & $7 \%$ & $46 \%$ & $44 \%$ \\
\hline $\begin{array}{l}\text { Trato del profesional } \\
\text { al momento de ser } \\
\text { atendido }\end{array}$ & $0 \%$ & $0 \%$ & $4 \%$ & $31 \%$ & $35 \%$ \\
\hline
\end{tabular}


CIENCIAMATRIA

Revista Interdisciplinaria de Humanidades, Educación, Ciencia y Tecnología

Año VII. Vol. VII. N¹2. Enero - Junio. 2021

Hecho el depósito de ley: pp201602FA4721

ISSN-L: 2542-3029; ISSN: 2610-802X

Universidad Nacional Experimental Francisco de Miranda (UNEFM). Santa Ana de Coro. Venezuela

Juan Andrés Palomeque-Cabrera; Jorge Edwin Ormaza-Andrade;

Guido Olivier Erazo-Álvarez

Además de los datos indicados anteriormente, con los datos tabulados por parte de las encuestas realizadas a los usuarios en términos generales existe aceptación del servicio brindado por parte de la Unidad de Matriculación del GAD de Azogues.

\section{PROPUESTA}

En la actualidad, las organizaciones empresariales e instituciones públicas tratan de incorporar cada vez más modelos de Responsabilidad Social, para obtener un impacto positivo.

Para cada segmento es idóneo identificar los aspectos claves y su vinculación directa con la responsabilidad social para el cual se ha definido los esquemas para conseguir el fin propuesto.

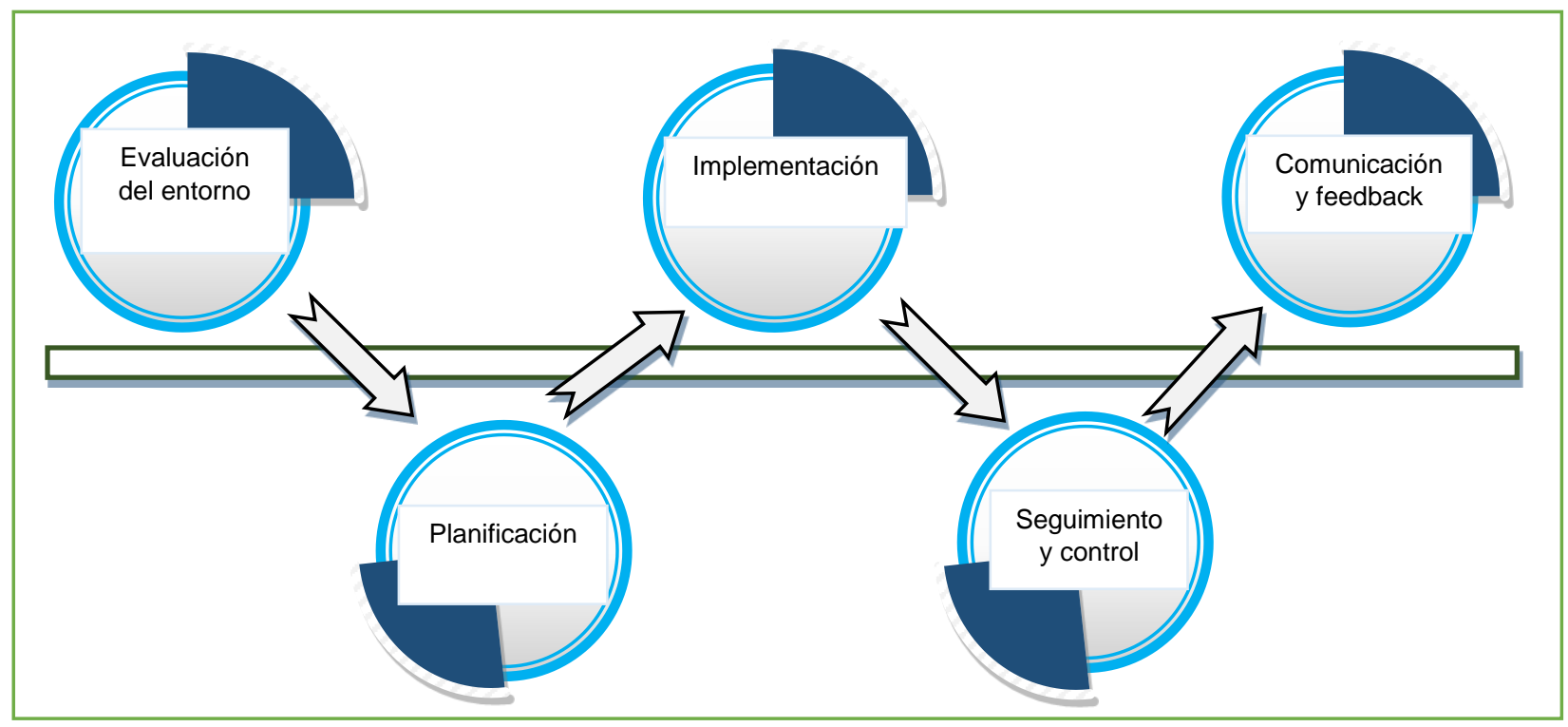

Figura 1. Esquema de la propuesta La Responsabilidad Social en la Unidad de Matriculación del GAD de Azogues. 


\section{CIENCIAMATRIA}

Revista Interdisciplinaria de Humanidades, Educación, Ciencia y Tecnología

Año VII. Vol. VII. N¹2. Enero - Junio. 2021

Hecho el depósito de ley: pp201602FA4721

ISSN-L: 2542-3029; ISSN: 2610-802X

Universidad Nacional Experimental Francisco de Miranda (UNEFM). Santa Ana de Coro. Venezuela

Juan Andrés Palomeque-Cabrera; Jorge Edwin Ormaza-Andrade;

Guido Olivier Erazo-Álvarez

Para la gestión de la Responsabilidad Social se seguirá los principios básicos en cuanto respecta a la transparencia, mejora continua y ética social. Todo aquello con la finalidad de dar a conocer como se encuentra el servicio ante la ciudadanía.

\section{Evaluación del entorno}

En este punto se trabajará recogiendo información de la institución e información externa que vendría a ser por parte de las encuestas a los usuarios del servicio de Matriculación del GAD de Azogues para poder realizar un análisis base:

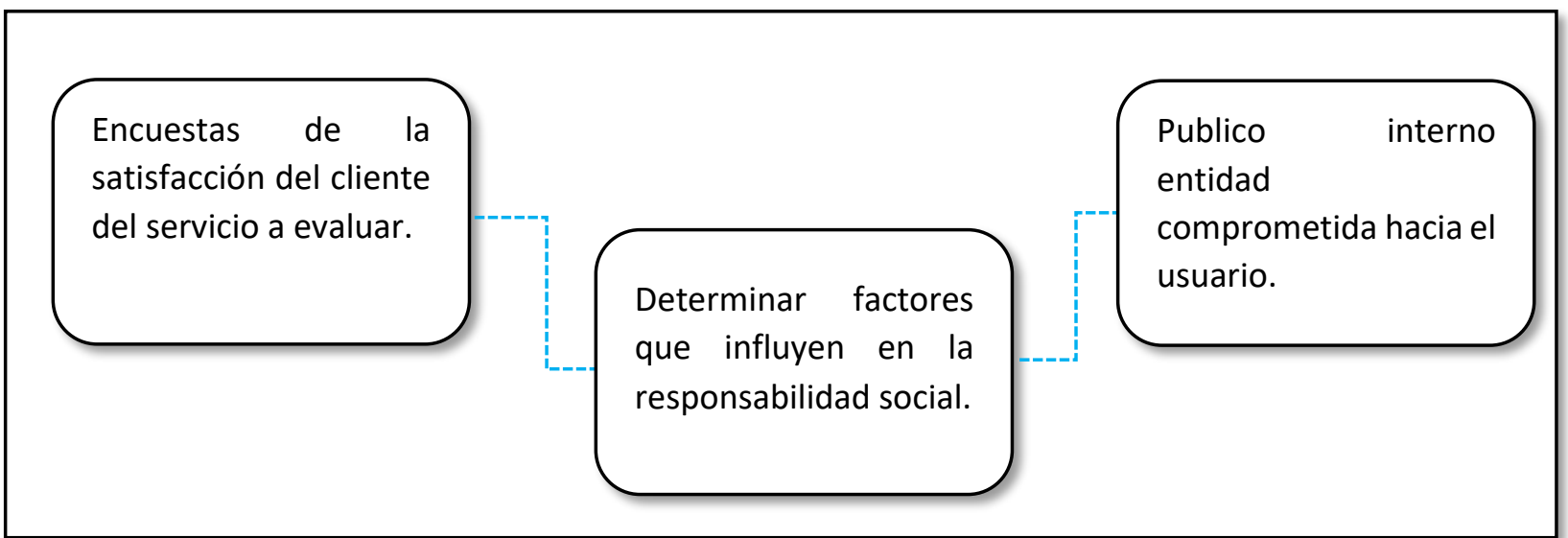

Figura 2. Evaluación del entorno.

\section{Planificación.}

Con los resultados obtenidos en la evaluación del entorno, contando con los recursos existentes, las circunstancias suscitadas y las necesidades detectadas se fundamenta el plan a realizar: 


\section{CIENCIAMATRIA}

Revista Interdisciplinaria de Humanidades, Educación, Ciencia y Tecnología

Año VII. Vol. VII. N¹2. Enero - Junio. 2021

Hecho el depósito de ley: pp201602FA4721

ISSN-L: 2542-3029; ISSN: 2610-802X

Universidad Nacional Experimental Francisco de Miranda (UNEFM). Santa Ana de Coro. Venezuela

Juan Andrés Palomeque-Cabrera; Jorge Edwin Ormaza-Andrade;

Guido Olivier Erazo-Álvarez

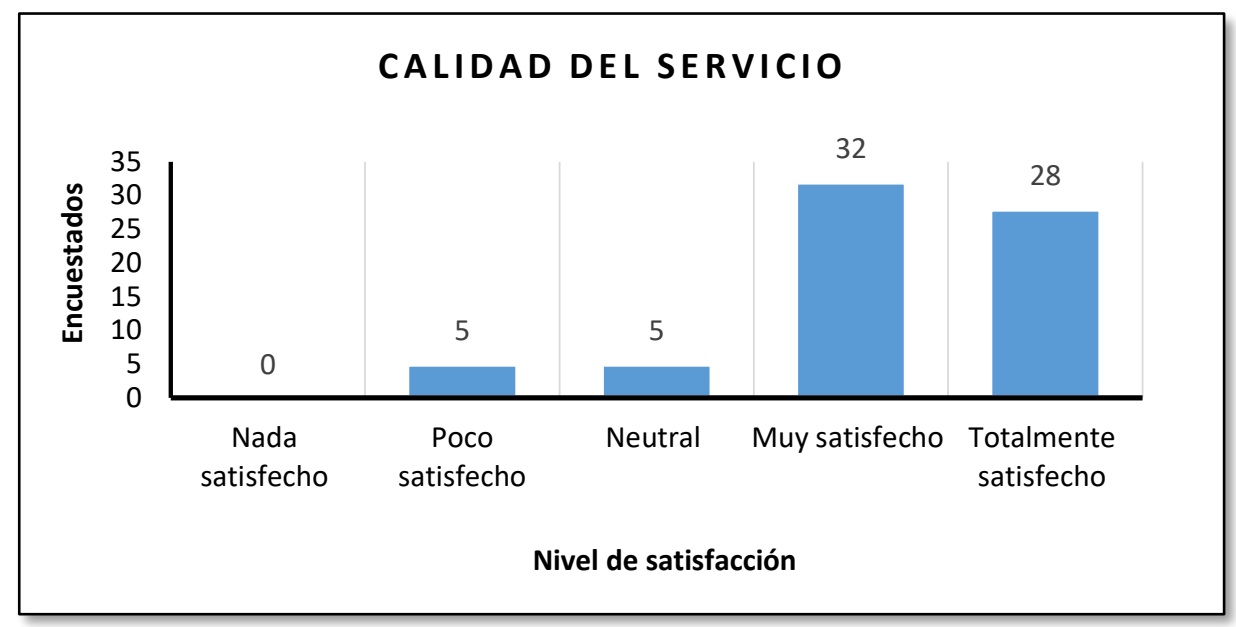

Figura 3. Medición de la calidad del servicio de Matriculación Vehicular.

1. Diagnóstico de los resultados de las encuestas.

2. Plan de mejora en los aspectos negativos de los resultados obtenidos de las encuestas.

3. Propuesta encaminada hacia la satisfacción del cliente.

\section{Implementación.}

Según la estrategia se trata establecer planes de mejora a futuro con los recursos existentes:

1. Charlas y capacitaciones acerca de la atención al cliente.

2. Motivación a los empleados. 


\section{CIENCIAMATRIA}

Revista Interdisciplinaria de Humanidades, Educación, Ciencia y Tecnología

Año VII. Vol. VII. N¹2. Enero - Junio. 2021

Hecho el depósito de ley: pp201602FA4721

ISSN-L: 2542-3029; ISSN: 2610-802X

Universidad Nacional Experimental Francisco de Miranda (UNEFM). Santa Ana de Coro. Venezuela

Juan Andrés Palomeque-Cabrera; Jorge Edwin Ormaza-Andrade;

Guido Olivier Erazo-Álvarez

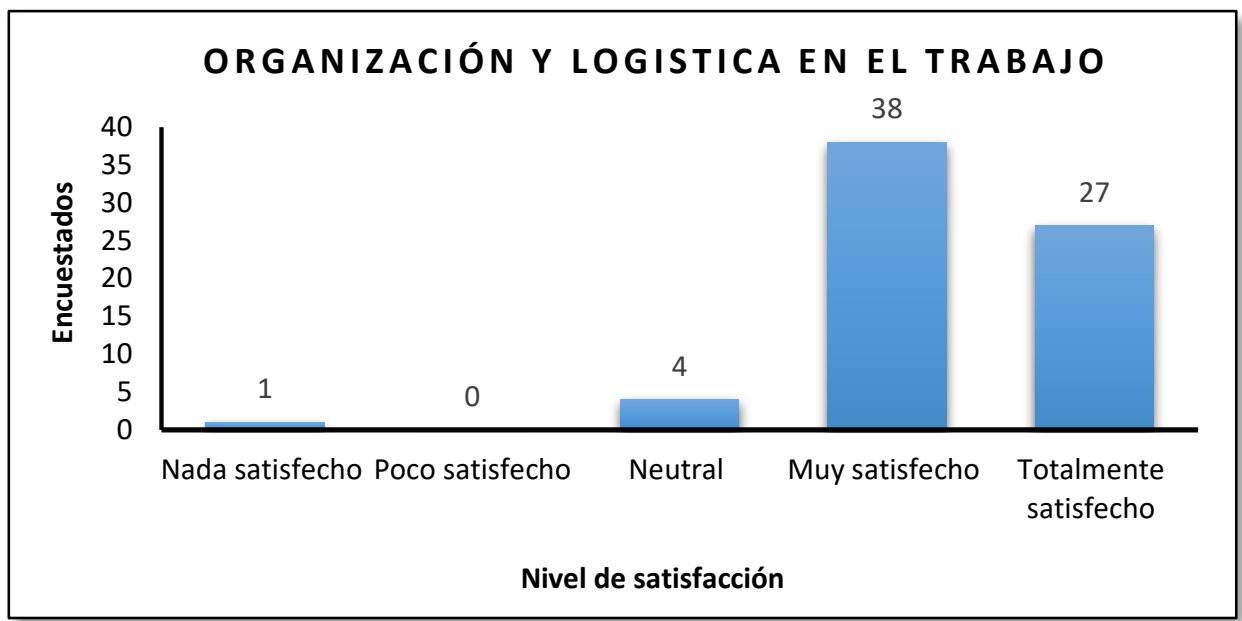

Figura 4. Medición de la organización en el trabajo.

\section{Seguimiento y control}

Se trata de poder medir resultados y de la implementación de la estrategia:

1. Cumplir con las expectativas de los usuarios.

2. Encuestas trimestrales.

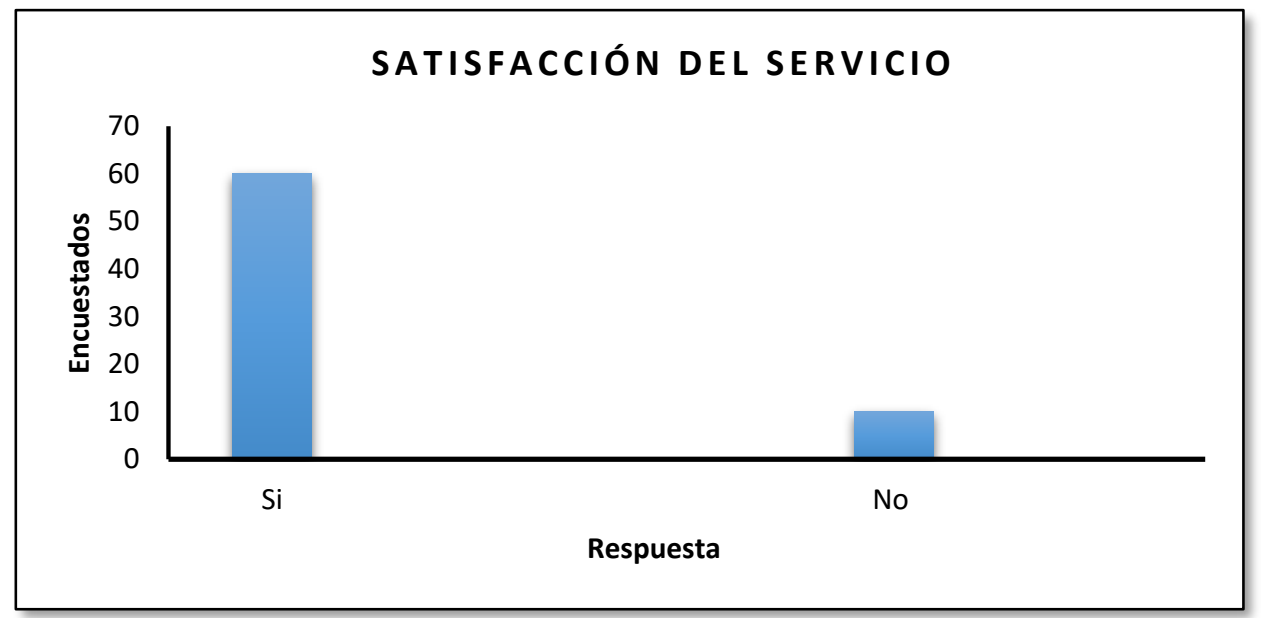

Figura 5. Satisfacción del servicio. 
CIENCIAMATRIA

Revista Interdisciplinaria de Humanidades, Educación, Ciencia y Tecnología

Año VII. Vol. VII. N¹2. Enero - Junio. 2021

Hecho el depósito de ley: pp201602FA4721

ISSN-L: 2542-3029; ISSN: 2610-802X

Universidad Nacional Experimental Francisco de Miranda (UNEFM). Santa Ana de Coro. Venezuela

Juan Andrés Palomeque-Cabrera; Jorge Edwin Ormaza-Andrade;

Guido Olivier Erazo-Álvarez

\section{Comunicación y feedback.}

Lo que se pretende en este punto, es la constante retroalimentación y comunicación con los usuarios que reciben el servicio para estar al pendiente de las nuevas situaciones que se pueden suscitar realizándola de con lo siguiente:

1. Campaña acerca del servicio brindado.

2. Casillero de quejas.

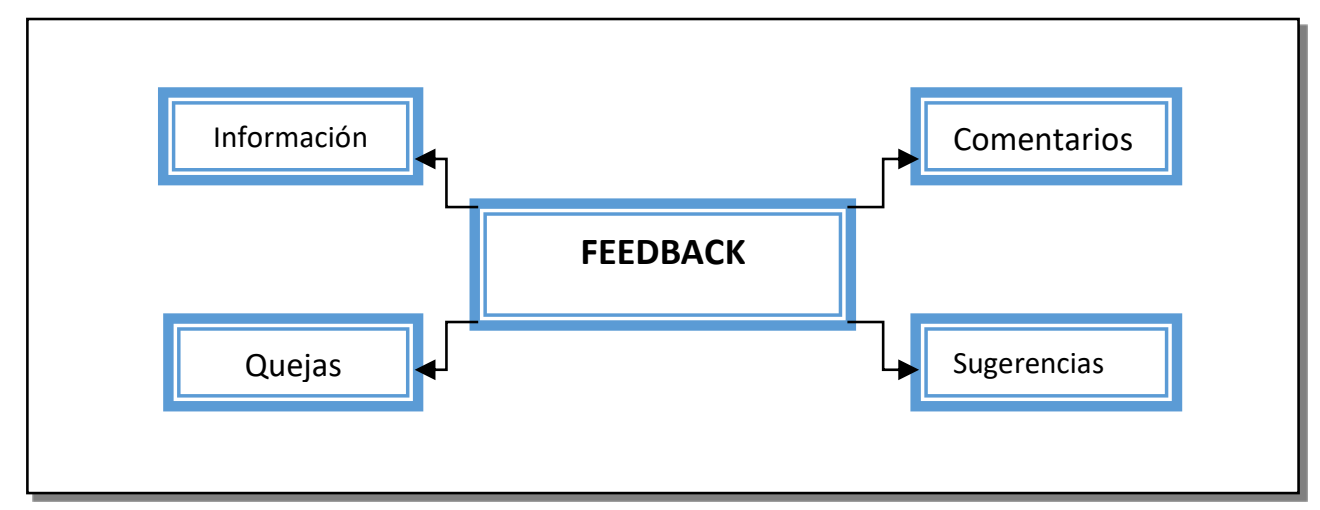

Figura 6. Satisfacción del servicio.

En definitiva, enfocamos el plan de responsabilidad social para la Unidad de Matriculación Vehicular del GAD de Azogues con el fin de lograr un mejor servicio en el ámbito laboral, ético y social para con la ciudadanía, además de comunicar a la ciudadanía y a los usuarios del servicio que se están haciendo acciones para su mejora, continúa velando por la colectividad.

\section{CONCLUSIONES}

De parte de la responsabilidad social de las entidades públicas lo que se pretende es brindar un trato justo, equitativo y de calidad al preocuparse por sus mandantes no únicamente realizar la rendición de cuentas sino de mejorar los servicios públicos puesto que estos hacen uso centenares de personas diariamente. Por medio de los estudios realizados enfocados a la responsabilidad social por parte de las entidades públicas lo 


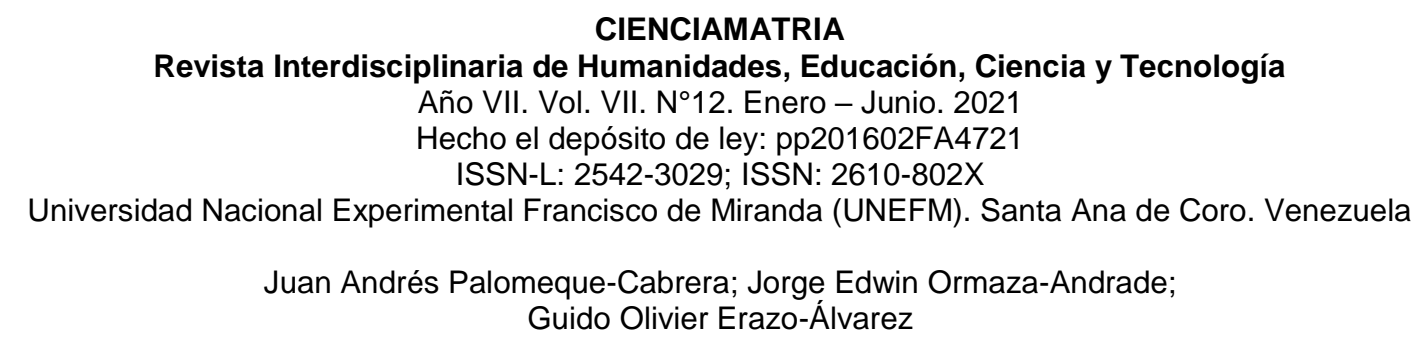

que se pretendió es mostrar ante la ciudadanía y a los mismos que son afines de la creación de los distintos servicios que se ofrecen, que la Unidad de Matriculación del GAD de Azogues se preocupa por la colectividad siendo socialmente responsables, realizando estudios de medición de la satisfacción del cliente por el servicio que se brinda fomentando un entramado positivo ante la ciudadanía obteniendo resultados alentadores con cerca del $85 \%$ de satisfacción del servicio brindado, además de ello queda una propuesta de puntos a mejorar en el servicio para estar siempre velando por el mismo, estar en constante contacto puesto que la meta de toda institución es la mejora continua.

\section{REFERENCIAS CONSULTADAS}

Aceves-López, J., \& Solórzano-Barrera, G. (2013). Importancia de la calidad del servicio al cliente para el funcionamiento de las empresas. El Buzón de Pacioli, 4-13. https://n9.cl/tnb5x

Albrecht, K. (1992). Servicio al cliente interno: como solucionar crisis de liderazgo en la gerencia intermedia. España : PAIDOS IBERICA.

Alvarado-Valencia, J. A., \& Trespalacios-Leal, M. C. (2016). Entendiendo la evaluación de la espera desde la psicología del consumidor: efectos de las expectativas y los llenadores de tiempo. Innovar, 26(62), 57-68. https://doi.org/10.15446/innovar.v26n62.59388

Andrea-Trujillo, M., \& Vélez-Bedoya, R. (2006). Responsabilidad ambiental como estrategia para la perdurabilidad empresarial. Bogota: Universidad Empresa.

Aniorte, N. (2020). Servicios de Calidad. Recuperado de https://n9.cl/kp6yv

Bertram, D. (2008). Topic Report. Recuperado https://n9.cl/pyctl

Block, R. A., Hancock, P. A., \& Zakay, D. (2000). Sex differences in duration judgments: a meta-analytic review. Memory \& cognition, 28(8), 1333-1346. https://doi.org/10.3758/bf03211834 
Calle-Calderón, K., Erazo-Álvarez, J., \& Narváez-Zurita, C. (2020). Marketing digital y estrategias online en el sector de fabricación de muebles de madera. Revista Arbitrada Interdisciplinaria Koinonía, 5(10), 339-369. http://dx.doi.org/10.35381/r.k.v5i10.698

Correa-Jaramillo, J. G. (2007). Evolución histórica de los conceptos de responsabilidad social empresarial y balance social. Semestre Económico, 10(20), 87-102.

Cuevas-Moreno, R., \& Rodrigues-Minor, R. (2017). Responsabilidad social y ética profesional en la gestión de la administración pública y empresarial. Pensamiento \& Gestión, (42),1-25.

Dutka, A., \& Mazia, A. (1994). Manual de AMA para la satisfacción del cliente. Buenos Aires: Ediciones Granica S.A.

Efiempresa. (2017). Calidad de servicio y Satisfacción del cliente. Recuperado de https://n9.cl/4mxf8

Fernández, P. D. (2016). El ciudadano como accionista: la RS en las empresas públicas. Recuperado de https://n9.cl/dyupk

Gonzáles-Sotelo, C. I., \& Naranjo-Africano, G. S. (2017). La responsabilidad institucional pública: un análisis para el mejoramiento de la gestión y la gerencia en la administración pública. Revista CIFE: Lecturas De Economía Social, 17(27), 245296. https://doi.org/10.15332/s0124-3551.2015.0027.07

Ilustre Municipalidad de Azogues Alcaldia . (2020). Unidad de Matriculación del GAD de Azogues . Azogues: Colad.

Instituto Ethos de Empresas e Responsabilidad Social. (2011). Indicadores Ethos de Responsabilidad Social Empresarial. Sao Paulo: Benjamin S Goncalves.

Lazo-Torres, N., Erazo-Álvarez, J., \& Narváez-Zurita, C. (2019). El Balanced Scorecard como herramienta de control interno en el sector Manufacturero. Revista Arbitrada Interdisciplinaria Koinonía, 4(1), 125-152. http://dx.doi.org/10.35381/r.k.v4i1.374 


\section{CIENCIAMATRIA}

Revista Interdisciplinaria de Humanidades, Educación, Ciencia y Tecnología

Año VII. Vol. VII. N¹2. Enero - Junio. 2021

Hecho el depósito de ley: pp201602FA4721

ISSN-L: 2542-3029; ISSN: 2610-802X

Universidad Nacional Experimental Francisco de Miranda (UNEFM). Santa Ana de Coro. Venezuela

Juan Andrés Palomeque-Cabrera; Jorge Edwin Ormaza-Andrade;

Guido Olivier Erazo-Álvarez

Ministerio del Trabajo. (2020). Satisfacción de la calidad del servicio y de la atención del servidor público. Quito: Ministerio del Trabajo.

Moreno, D., \& Bernal, M. (2014). Importancia del cliente interno y externo en las organizaciones. BOGOTÁ: Universidad Militar Nueva Granada.

Pérez, M. (2020). Responsabilidad. Recuperado de https://n9.cl/awyo

Pérez-Espinoza, M. J., Espinoza-Carrión, C., \& Peralta-Mocha, B. (2016). La responsabilidad social empresarial y su enfoque ambiental: una vision sostenible a futuro. Revista Universidad y Sociedad, 8(3), 169-178.

Prieto, R. (2015). Los Stakeholders y la Responsabilidad Social Corporativa. En El Gobierno Corporativo en Iberoamérica (p. 477-522). Madrid: CYAN, Proyectos Editoriales S.A. https://n9.cl/etvz

Real Academia Española RAE. (2001). Responsabilidad. Recuperado de https://n9.cl/qcr8

Ruiz-Rico Ruiz, C. (2014). Responsabilidad social pública y calidad democrática. Derecho y cambio social, 11(37), 1-17.

Thompson, I. (2005). La satisfacción del cliente. https://n9.cl/qjurr

Vartuli, A. (2008). World Documents. Recuperado de https://n9.cl/04

Zagal, P. M. (2010). Medición y control del nivel de satisfaccion de los clientes en un supermercado . Piura: Universidad De Piura. 